\title{
Topological characterization of the transition from laminar regime to fully developed turbulence in the resistive pressure-gradient-driven turbulence model
}

\author{
L. Garcia* and B. A. Carreras \\ Departamento de Física, Universidad Carlos III de Madrid, 28911 Leganés, Madrid, Spain
}

I. Llerena

Departament d'Àlgebra i Geometria, Facultat de Matemàtiques, Universitat de Barcelona, Barcelona, Spain

I. Calvo

Laboratorio Nacional de Fusión, Asociación EURATOM-CIEMAT, 28040 Madrid, Spain

\begin{abstract}
For the resistive pressure-gradient-driven turbulence model, the transition from laminar regime to fully developed turbulence is not simple and goes through several phases. For low values of the plasma parameter $\beta$, a single quasicoherent structure forms. As $\beta$ increases, several of these structures may emerge and in turn take the dominant role. Finally, at high $\beta$, fully developed turbulence with a broad spectrum is established. A suitable characterization of this transition can be given in terms of topological properties of the flow. Here, we analyze these properties that provide an understanding of the turbulence-induced transport and give a measure of the breaking of the homogeneity of the turbulence. To this end, an approach is developed that allows discriminating between topological properties of plasma turbulence flows that are relevant to the transport dynamics and the ones that are not. This is done using computational homology tools and leads to a faster convergence of numerical results for a fixed level of resolution than previously presented in Phys. Rev. E 78, 066402 (2008).
\end{abstract}

DOI: 10.1103/PhysRevE.80.046410

PACS number(s): 52.35.Ra, 52.65.Kj, 47.27.-i

\section{INTRODUCTION}

In recent years, numerical studies of tracers in turbulent plasma models have revealed the nondiffusive nature of the particle tracer transport at least in some parameter range [1-7]. All these studies have looked at the different properties of the particle tracer distribution, their velocity distribution, and the long-range correlations. From these studies, it has been possible to determine the types of fractional differential equations [8] that could describe the transport of those particle tracers.

What has not yet been done is a study of the properties of the underlying turbulence that lead to these features of the particle tracer transport. In this paper, we will take a first step in this direction by using quantitative measures of the topological properties of the turbulent flow to connect with the properties of the distribution function of the particle tracers in these turbulent flows.

In the present study, we use the resistive pressuregradient-driven turbulence model. In Ref. [9] and for this model, the transition from laminar regime to fully developed turbulence was described. As the plasma $\beta$ increases, the turbulence and the induced transport go through several phases. For low values of the plasma parameter $\beta$, a single quasicoherent structure forms $[2,9]$ and a single toroidal mode dominates the spectrum. As $\beta$ increases, the turbulence becomes highly intermittent because several of these quasicoherent structures may emerge and in turn take the dominant role. Finally, at high $\beta$, fully developed turbulence with a broad spectrum is established.

\footnotetext{
*1garcia@fis.uc3m.es
}

All the phases of this transition were identified $[2,9,10]$ through spectral analysis and visualization of the flow structures. However, the topological changes were not properly characterized by any quantitative diagnostics of the topology properties of the flow, which seems to be the most appropriate way of characterizing the transition.

In this paper, we do this quantitative evaluation of the topological properties of the flows using and improving the topological techniques that we have introduced in Ref. [11]. The approach proposed in that paper consists on the numerical determination of the Betti numbers of the isosurfaces of the velocity stream function.

For the complicated flow structures obtained in numerical calculations of plasma turbulence, the determination of the Betti numbers is far from trivial. In Ref. [11] and in what follows, we use the software package provided by the Computational Homology Project (CHomP) [12] to carry out this determination. The CHomp group has developed many numerical tools. A description of such tools and some examples of applications can be found in the CHomP website [12]. Reference [13] gives a systematic computational approach to the homology of cubical sets, which is the approach that we follow here.

In our initial numerical studies [11], two main issues have emerged. One is a resolution limitation, which was only partially addressed in Ref. [11]. Namely, for high resolution calculations, the memory limit set in the CHomP software does not allow us an accurate determination of the Betti numbers of the topological structure of the flows from our high $\beta$ calculations. Here $\beta$ is the ratio of the plasma pressure to the magnetic pressure.

The second issue is that not all topological structures are equally relevant to the transport physics, which is the goal of 
our studies. For instance, the existence of filaments in the flow structure that twist around the whole toroidal plasma and create large-scale cycles plays an important role in the pseudochaotic behavior of the tracer particles [2,10,14]. Furthermore, such filaments break the homogeneity and isotropy of the plasma turbulence modifying as a consequence the turbulence-induced transport. Small very localized cycles, which are not relevant to the transport, may also exist. Some of those small cycles are simply spurious, originating from a lack of numerical resolution. However, both the large-scale cycles and the small-scale cycles contribute to the value of the Betti numbers of the velocity stream function isosurfaces.

In this paper, we develop an approach that allows the determination of an upper bound to the number of the largescale cycles and the distribution of large-scale connected components in the plasma flow. This approach, in isolating the large-scale cycles from almost all small cycles, gives a determination of their number that converges very fast. The convergence is faster than the convergence of the total number of cycles for a given level of resolution of the flow structure. We also determine a way of identifying the large-scale components, the ones that do not depend on the numerical resolution level. The distribution of these components is equivalent to the eddy-size distribution and therefore relevant to the transport physics.

The number of large-scale connected components of the flow and the number of large-scale cycles permit a quantitative characterization of the transition from laminar regime to fully developed turbulence. They also provide the basis for understanding the transport properties in these phases and open a way of connecting turbulence and induced transport. We also show that even in the high $\beta$ case, when graphical representations of the flow seem to indicate that the turbulence is relatively homogeneous, there are still large flow structures of complex topology. These structures effectively break the homogeneity and isotropy of the turbulence.

The rest of the paper is organized as follows. In Sec. II, we introduce the topological parameters that will be used in the present analysis. In Sec. III, the dynamical evolution of these parameters is analyzed for different values of the plasma parameter $\beta$. A more detailed analysis of the flow structures from the perspective of their radial extent is done in Sec. IV. In Sec. V, we show how changes in the flow topology affect the transport of tracers. Finally, in Sec.VI, the conclusions of this paper are given.

\section{TOPOLOGICAL PARAMETERS OF THE FLOW STRUCTURES}

Our starting point is the topological analysis of the plasma flows. We center our analysis on the velocity stream function $\Phi$. The plasma flow velocity, $\mathbf{V}_{\perp}$, is given in terms of the velocity stream function, $\Phi$, by

$$
\mathbf{V}_{\perp}=\nabla \Phi \times \mathbf{b} .
$$

In this expression, $\mathbf{b}$ is a unit vector in the direction of the magnetic field. The velocity stream function is directly related to the electrostatic potential of the plasma $\phi$ by $\Phi=$ $-\phi / B$, where $B$ is the intensity of the magnetic field. The magnetic field is assumed to be constant in time because we are studying electrostatic turbulence. Therefore, all the information on the structure of the velocity field is contained in the velocity stream function. We use $(\rho, \theta, \zeta)$ as the coordinates in toroidal geometry. At a fixed time $t$, we define a flow structure as the set of points such that $\Phi(\rho, \theta, \zeta, t) \geq \bar{\Phi}_{0}$, where $\bar{\Phi}_{0}$ is a constant. We define $\Phi_{0}=\bar{\Phi}_{0} / \max (\Phi)$, with $\max (\Phi)$ being the maximum value $\Phi$ at time $t$. Therefore, $\Phi_{0}$ gives a fraction of the maximum value of $\Phi$ and $0 \leq \Phi_{0} \leq 1$.

In general, either in numerical calculations or in analytical modeling, we use a representation of $\Phi$ in Fourier series

$$
\Phi(\rho, \theta, \zeta, t)=\sum_{m, n} \Phi_{m n}(\rho, t) \sin (m \theta+n \zeta) .
$$

Here, $m$ and $n$ are the poloidal and toroidal mode numbers, respectively.

A first approach in characterizing the topology of a flow structure is to determine its Betti numbers. For structures in $\mathrm{R}^{3}$, only three of these topological invariants are nonzero [15]. We denote them by $b_{0}, b_{1}$ and $b_{2} . b_{0}$ is the number of arc-connected components of the structure, $b_{1}$ is the number of (independent) noncontractible loops, and $b_{2}$ is the number of voids.

As described in Ref. [11], we use the software package provided by the Computational Homology Project (CHomP) [12], that computes the Betti numbers of cubic spaces, i.e., spaces that are the union of a finite number of cubes with unit side length and vertices with integer coordinates [13]. The data files used by the CHomP code are in the form of a list of cubes with integer coordinates. So, to analyze the topological structure of the flow, the first thing to do is to construct a cubical space that approximates as much as possible the flow structure,

$$
\begin{aligned}
\{(\rho, \theta, \zeta) \mid \Phi(\rho, \theta, \zeta) & \geq \bar{\Phi}_{0}, 0 \leq \rho \leq 1,0 \leq \theta \leq 2 \pi, 0 \leq \zeta \\
& \leq 2 \pi\} .
\end{aligned}
$$

For a structure in $\mathbb{R}^{3}$, the maximum number of cubes that can be used by the CHomP code is $2.6 \times 10^{8}$. In Ref. [11], we considered several ways of choosing the cubes to cover the flow structure in an optimal fashion and to minimize the number of cubes needed. In building the cubical space we choose $N_{\rho}=N_{\theta}=2 N_{\zeta}$, where $N_{\rho}, N_{\theta}$ and $N_{\zeta}$ are the number of cubes in the radial, poloidal and toroidal direction, respectively. Here, we follow the optimization method described in Ref. [11]. In order to work with integer coordinates $\left(\rho_{1}, \theta_{1}, \zeta_{1}\right)$, we take the structure

$$
\begin{aligned}
& \left\{\left(\rho_{1}, \theta_{1}, \zeta_{1}\right) \mid \Phi\left(\frac{\rho_{1}}{N_{\rho}}, \frac{2 \pi \theta_{1}}{N_{\theta}}, \frac{2 \pi \zeta_{1}}{N_{\zeta}}\right) \geq \bar{\Phi}_{0}, 0 \leq \rho_{1} \leq N_{\rho}, 0\right. \\
& \left.\leq \theta_{1} \leq N_{\theta}, 0 \leq \zeta_{1} \leq N_{\zeta}\right\}
\end{aligned}
$$

This structure has the same topology as the one given in Eq. (3). 


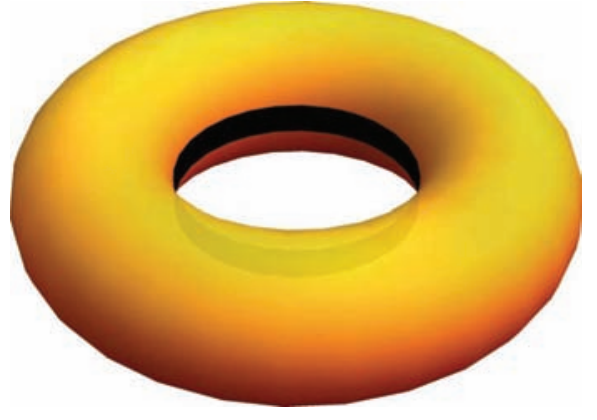

FIG. 1. (Color online) Torus showing a cut along the poloidal angle $\theta=0$.

The two relevant measures that we want to use are the number of connected components and the number of cycles. However, not all cycles and components have the same relevance to the physics of the plasma. Small cycles in the flow structure matter very little. These small cycles are not always a property of the flows, but, in most cases, are the result of the limitations in numerical resolution. The cycles we are interested in are the large-scale cycles that are formed by flow filaments turning all the way around the torus. In the Appendix, we give an approach to measure the number of large-scale cycles, $c_{1}$.

Following the notation of the Appendix, let us call $X$ our structure and $A$ the same structure after we cut it at $\theta=\pi$. The number of large-scale cycles is given by Eq. (A10),

$$
c_{1}=b_{1}[X, A]-b_{0}[A]+b_{0}[X] .
$$

Here, $b_{1}[X, A]$ is the rank of the relative homology group $H_{1}(X, A)$ of the pair $(X, A)$, and $b_{0}[X], b_{0}[A]$ are the zeroth Betti numbers of the sets $X$ and $A$, respectively.

From the excision theorem [15], we know that the rank of the relative homology, $b_{1}[X, A]$, can be calculated using subsets of $X$ and $A$ that are around the region of the $\theta=\pi$ cut, as indicated in Fig. 1. That implies that the cubical set used as data input can be relatively small and we do not reach the limit set by the CHomP. We use the CHomP code to calculate $b_{1}[X, A]$ and we can reach without problem global resolutions of $4800 \times 4800 \times 2400$ cubes, which is the resolution of the numerical results that we are analyzing.

The number of large-scale cycles $c_{1}$ converges very fast and in all the calculations presented here, we have wellconverged results for this parameter. With respect to the connected components, we have a similar situation to the cycles. There are some very small components in the cubical sets that are related to the limited resolution of the system and irrelevant to the physics. In order to detail information on the size distribution of the components and to be able to handle larger cubical sets than the CHomP code, we have built a solver to calculate them.

The way we calculate $b_{0}$ is by radial layers. We start at the smallest radius and calculate $b_{0}$ by adding a radial layer of cubes at the time. In this way, we can define $b_{0}(r)$, which is the value of $b_{0}$ for the structure $\Phi(\rho, \theta, \zeta, t) \geq \bar{\Phi}_{0}$ with $0 \leq \rho \leq r$. Therefore, we not only calculate the global $b_{0}$, but we also see how $b_{0}$ changes with radius. Furthermore, for

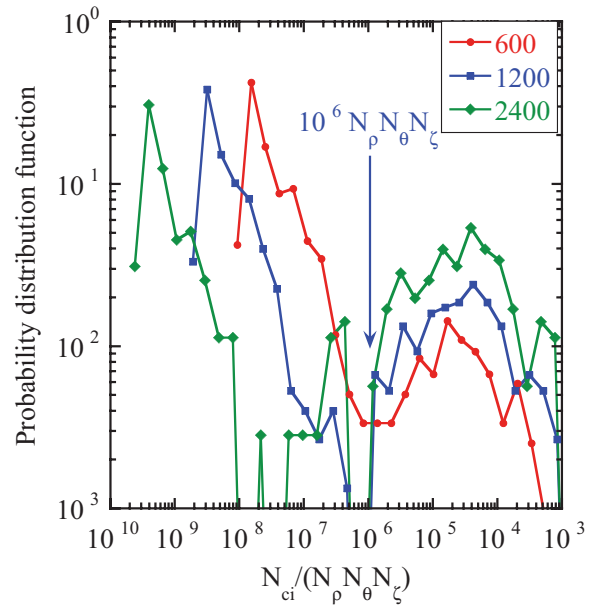

FIG. 2. (Color online) Probability distribution of the size of the conected components for $\beta_{0}=0.01$ and three levels of resolution, $N_{\rho}=600,1200$, and 2400 .

each component we calculate its size by the number of cubes that it contains and its radial extent. This provides us with additional information on the structure of the flow.

The size distribution of the components is not uniform. For the numerical results of the resistive pressure-gradientdriven turbulence, this distribution has two peaks: a large and narrow peak at the smaller end of the range of sizes and a broader peak at the other end, as can be seen in Fig. 2. If the size of the connected components is normalized to the total number of cubes, $N_{\rho} \times N_{\theta} \times N_{\zeta}$, then we have a measure of the size of the component, which is independent of the resolution level. Using this normalization, we can see that the peak of the size distribution at the smallest sizes moves downwards as the resolution increases and its height decreases. This indicates that the components corresponding to this peak depend on the resolution and they are not real components of the flow structure. However, the second peak of the distribution remains invariant as the resolution changes. Therefore, these components in the second peak of the distribution are the real components of the flow structure.

We define a reference size, $N_{r e f}=10^{-6} N_{\rho} \times N_{\theta} \times N_{\zeta}$. This size corresponds to the gap between the two peaks in the distribution. We select the components such that $N_{c i}>N_{\text {ref }}$ as the relevant ones for the flow structure. Here, $N_{c i}$ is the number of cubes of the component $i$. In analogy to the cycles, we will call these components large-scale components and their number is $n_{0}$.

In summary, the two measures that we use in the diagnosis of the flow structures are the number of large-scale connected components $n_{0}$ and the number of large-scale cycles, $c_{1}$.

\section{DYNAMICAL EVOLUTION OF THE RESISTIVE PRESSURE-GRADIENT-DRIVEN TURBULENCE}

Using the reduced set of resistive MHD equations [16,17] in the electrostatic approximation, we have studied the time evolution of the turbulence in a steady state for different values of $\beta$. For the parameters of these calculations [18], the 


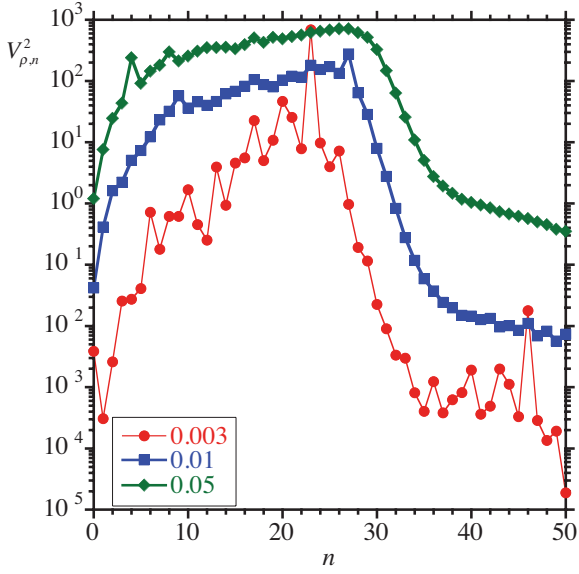

FIG. 3. (Color online) Toroidal mode spectrum of the square of the radial velocity for different values of $\beta$. These spectra have been calculated by averaging over the steady state regime of the resistive pressure-gradient-driven turbulence calculations from Ref. [18].

resistive ballooning modes are unstable above a $\beta$ value of 0.0025 . The nonlinear calculations have been done for four $\beta_{0}$ values, $0.003,0.006,0.01$, and 0.05 . Here, $\beta_{0}$ denotes the value of $\beta$ at the magnetic axis.

As already mentioned, for $\beta_{0}=0.003$, a single quasicoherent flow structure forms $[2,10]$ with a single toroidal mode $n=N$ dominating the $\Phi$-spectrum, Eq. (2). This can be seen in Fig. 3, where we show the toroidal mode spectrum of the radial velocity for different values of $\beta_{0}$. The instantaneous spectrum is obtained from the expansion (2) by

$$
V_{\rho, n}^{2}=\frac{1}{2} \sum_{m} m^{2} \int_{0}^{1} \frac{\Phi_{m n}^{2}(\rho)}{\rho^{2}} d \rho .
$$

In Fig. 3, we have plotted the time-averaged spectra over the steady-state phase of the time evolution. Therefore, for $\beta_{0}=0.003$, we expect a flow structure of a single mode type, a single value of $n$, where filamentary eddies form at the singular surfaces, they twist around the torus and merge in the outer regions forming the so-called streamers due to the ballooning effect. An example for an $N=3$ structure is shown in Fig. 4. In this figure, we have used such a low- $N$ case just to be able to visualize the structure. For the numerical results and for $\beta_{0}=0.003$, the $N=23$ is the dominant structure. In

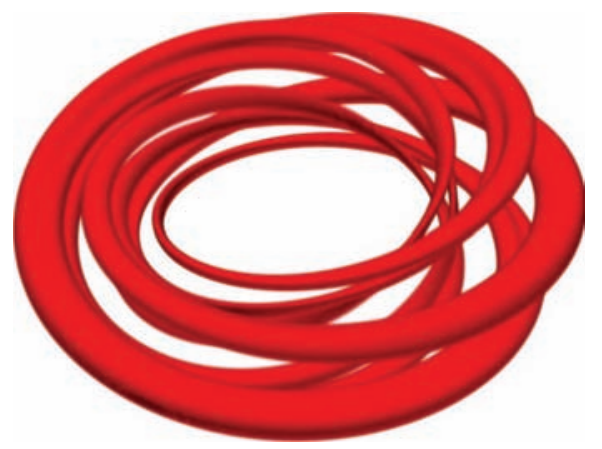

FIG. 4. (Color online) An example for an $N=3$ flow structure showing 3 filamentary eddies with $m=3,4$, and 5 .

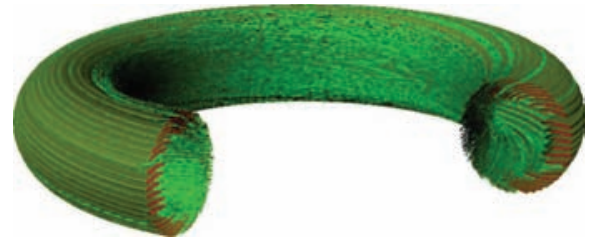

FIG. 5. (Color online) Contour plots of the function $\Phi$ for $\beta_{0}=0.003$ as obtained from the numerical calculations. The dominant structure is $N=23$.

Fig. 5, we have plotted the function $\Phi$ for this $\beta$ value. It is very hard to visually understand the structure.

The qualitative structure of $\Phi$ is such that at low values of $\Phi_{0}$ all filamentary eddies are thick enough to merge everywhere and there are practically no detectable large cycles. On the other hand, at values of $\Phi_{0}$ close to 1 , the filamentary eddies in the inner region of the torus have thinned so much that they break and the large-scale cycles disappear. Therefore, there is and optimal range of values of $\Phi_{0}$ in which we can explore most efficiently the topological structure.

The optimal range of values can be inferred from Fig. 6, where we have plotted the number of large-scale cycles as a function of $\Phi_{0}$ for the different $\beta$ values. From Fig. 6, we can see that the best range of values of $\Phi_{0}$ is between 0.1 and 0.3 .

In Fig. 7 and for $\Phi_{0}=0.1$, we can see the time evolution of the number of large-scale cycles for the different $\beta$ values. For the lowest $\beta$ value, $\beta_{0}=0.003$, an $N=23$ quasicoherent structure dominates the spectrum at all times. There is practically no change in time of the topological structure. As $\beta$ increases, different quasicoherent structures compete, this leads to intermittent behavior and oscillations of $c_{1}$ as shown for $\beta_{0}=0.006$. At higher $\beta$ values, the mean value and the time variation of $c_{1}$ decrease but its averaged value remains relatively high.

The values of $c_{1}$ are very well converged for a resolution of $N_{\rho}=4800$. In Fig. 8, we show an illustration of the convergence of $c_{1}$ with the number of cubes. We can see that even for $N_{\rho}=1200$ the values of $c_{1}$ are very close to their converged values.

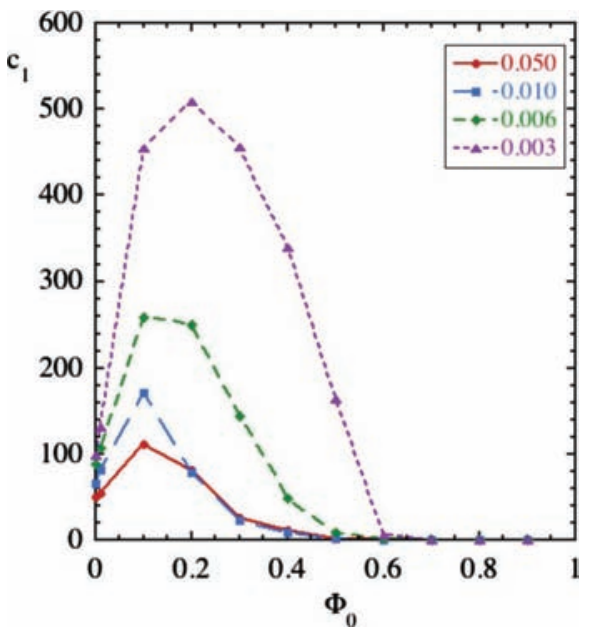

FIG. 6. (Color online) Number of large-scale cycles as a function of $\Phi_{0}$ at a given time for different $\beta_{0}$ values. 


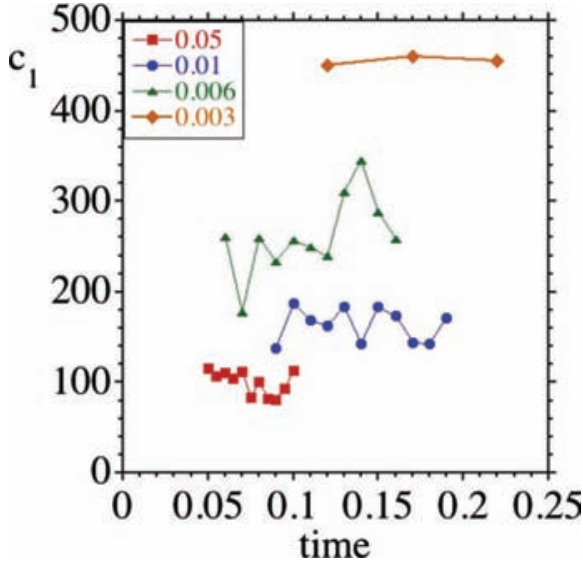

FIG. 7. (Color online) Time evolution of the number of largescale cycles for different $\beta_{0}$ values and for $\Phi_{0}=0.1$ during the steady-state regime of the resistive pressure-gradient-driven turbulence calculations from Ref. [18].

The time evolution of the large-scale components is illustrated in Fig. 9. In this figure, we have plotted $n_{0}$ as a function of time for $\Phi_{0}=0.3, N_{\rho}=1200$, and the same four $\beta_{0}$ values as in Fig. 7. The number of large-scale components oscillates in time more than $c_{1}$.

The values of $n_{0}$ are well converged as we change the number of cubes. An example is shown in Fig. 10. In this figure, we have plotted the number of large-scale components, $n_{0}$, as a function of $\Phi_{0}$ for $\beta_{0}=0.01$ and for the different levels of numerical resolution. The distributions for $N_{\rho}=1200$ and 2400 lie almost on top of each other. The one for 600 is slightly displaced. Therefore, for $N_{\rho}=1200$, the number of large-scale components is well converged. For low values of $\Phi_{0}$, the number of components increases as $\Phi_{0}$ increases because filaments start to break, but at large $\Phi_{0}$ they decrease because the flow structure becomes smaller and smaller.

We summarize in Fig. 11 how the topological parameters of the flow change with $\beta$. We have plotted the time average of $c_{1}$ and $n_{0}$ as functions of $\beta_{0}$. The error bars give the

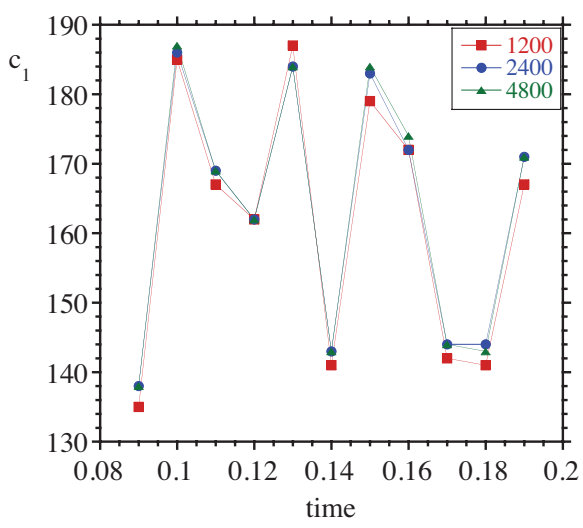

FIG. 8. (Color online) Convergence of the number of large-scale cycles, $c_{1}$, with varying resolution. The three cases plotted here are for $N_{\rho}=1200,2400$, and 4800 . The time evolution corresponds to the steady-state regime of the resistive pressure-gradient-driven turbulence calculations from Ref. [18] for $\beta_{0}=0.01$.

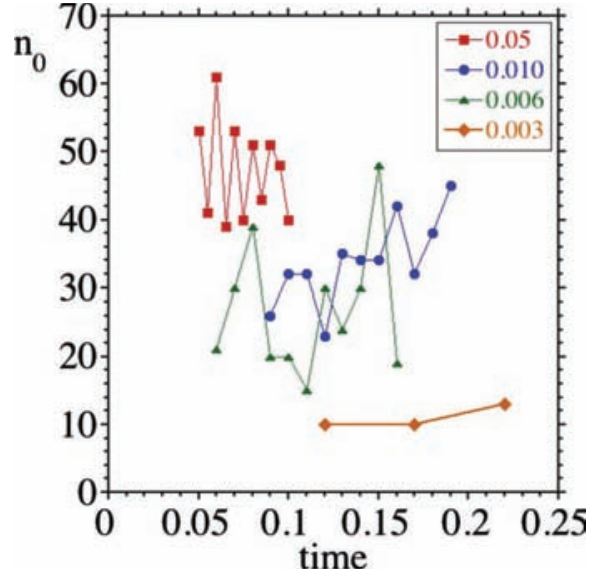

FIG. 9. (Color online) Time evolution of the large-scale components as a function of time for $\Phi_{0}=0.3, N_{\rho}=1200$, and the same four $\beta_{0}$ values as in Fig. 7.

standard deviation of these two quantities during the time evolution. The plot is done for $\Phi_{0}=0.1$. Above the instability threshold there is a sharp increase in $c_{1}$ and then as $\beta$ increases $c_{1}$ decreases. However, at the higher $\beta$ values, the decrease of $c_{1}$ is rather slow. On the other hand, $n_{0}$ is equal to 1 just above the threshold and jumps to higher values as $\beta$ increases.

\section{RADIAL EXTENT OF THE FLOW STRUCTURES}

To better understand the convergence of the number of large-scale components and their size distribution, we examine them in more detail. We will focus on the radial extent of the components because we are interested in relating them to the radial transport.

In all cases considered, there is always a large flow component. This component is well described at all levels of resolution and its size decreases with increasing $\Phi_{0}$. As could be expected, there is no convergence problem with this component when we vary the number of cubes.

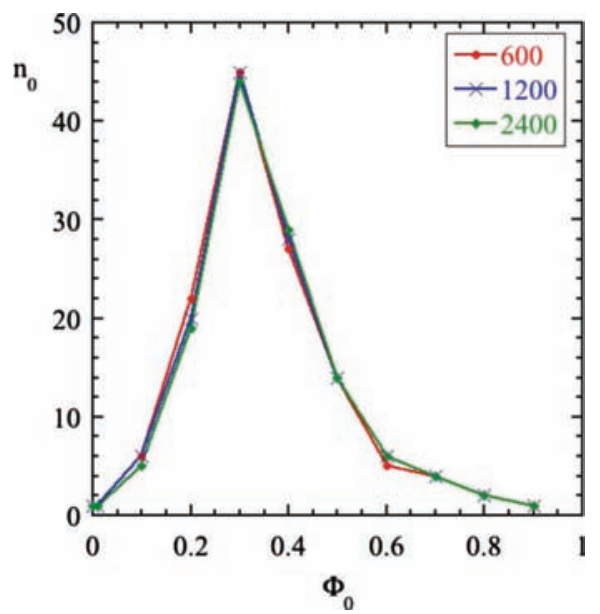

FIG. 10. (Color online) Number of large-scale components $n_{0}$ as a function of $\Phi_{0}$ for $\beta_{0}=0.01$ and for the different levels of resolution. 


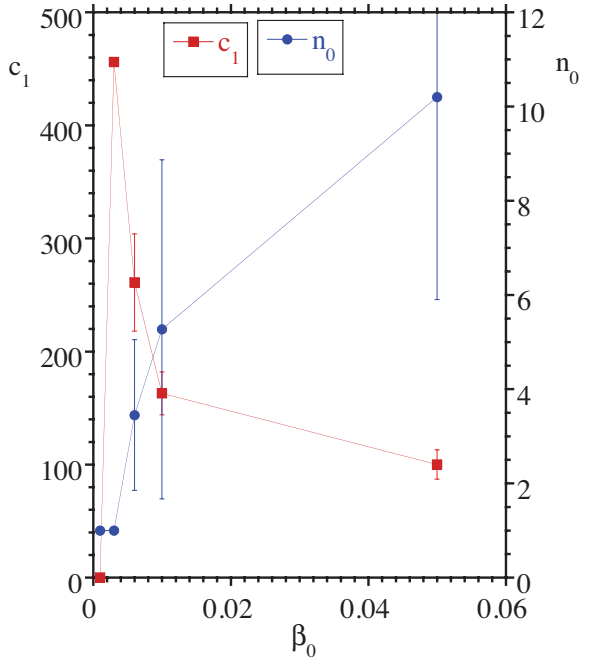

FIG. 11. (Color online) Time average of $c_{1}$ and $n_{0}$ as a function of $\beta_{0}$. The error bars give the standard deviation of these two quantities during the time evolution. The plot is done for $\Phi_{0}=0.1$ and the same nonlinear calculations and resolution levels as in Figs. 7 and 9.

Let us consider now the other large-scale components of the flow. In Fig. 12, we have plotted a set of the large-scale components for $\beta_{0}=0.01$ and for $\Phi_{0}=0.1$. We represent them as a function of radius by their radial extent, shown as a horizontal line. They are ordered by size, total number of cubes, from bottom to top. We show these components for three levels of resolution. We can see that the pattern for $N_{\rho}=1200$ and 2400 is practically the same. Only two components that were not connected for $N_{\rho}=1200$ merge in a single one for $N_{\rho}=2400$. This confirms that all large-scale components are well described for resolutions of the order of $N_{\rho}=1200$.

Even at the highest $\beta$ value considered here, $\beta_{0}=0.05$, there is a large connected component for a broad range of

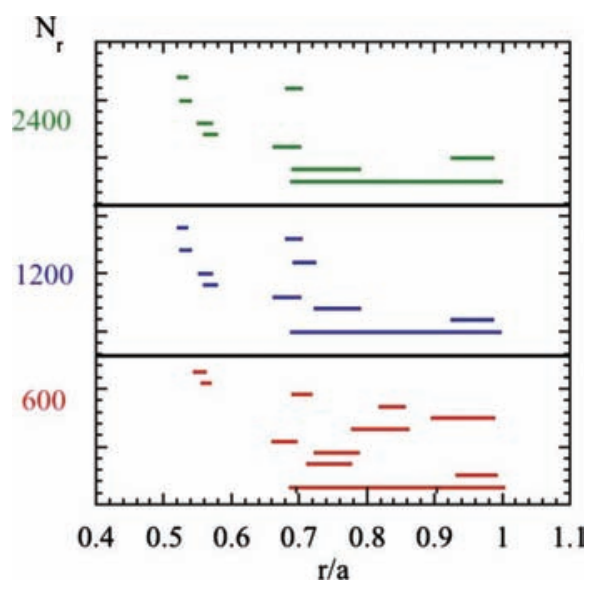

FIG. 12. (Color online) Large-scale components for $\beta_{0}=0.01$ and for $\Phi_{0}=0.1$. We represent them as a function of radius by their radial extent, shown as a horizontal line. They are ordered by size, total number of cubes, from bottom to top. We show these components for three levels of numerical resolution indicated by the different values of $N_{\rho}$.

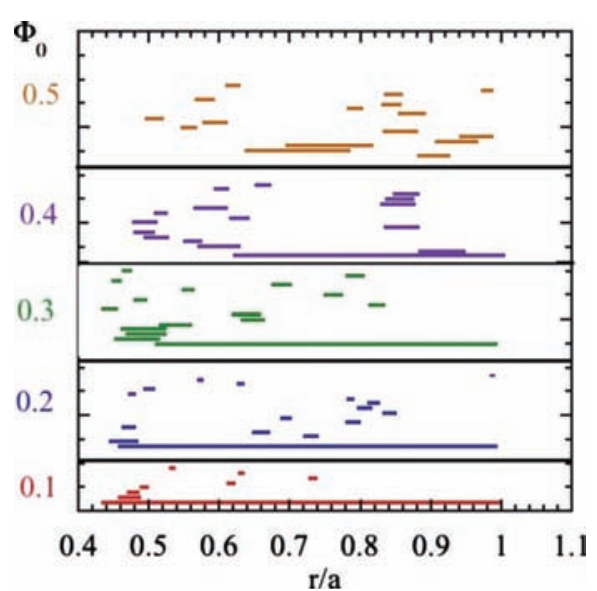

FIG. 13. (Color online) Large-scale components for $\beta_{0}=0.05$, $N_{\rho}=4800$ and different values of $\Phi_{0}$. This graph is limited to the largest 16 components.

values of $\Phi_{0}$ up to $\Phi_{0}=0.4$. For $\Phi_{0}=0.1$, the number of large-scale components is small, 8. The large component dominates most of the radial range; a few other components appear mostly inside and due to the breaking of some filaments. As we increase $\Phi_{0}$, we see a continuous breaking of the filaments in the inner region of the radius with the dominant component reducing its size from the inside. This can be seen in Fig. 13 where we have plotted the large-scale components for $\beta=0.05$ and different values of $\Phi_{0}$. In this figure, we have not plotted all the large-scale components in each case to avoid crowding too much the plot. We have limited the graph to the largest 16 components.

In Fig. 14, we give the number of large-scale components as a function of $\Phi_{0}$ for the four values of $\beta_{0}$ considered in this study. The values in this plot are the time averages and the error bars stand for the standard deviation. For low values of $\Phi_{0}$, there is a slight increase in the number of components as $\beta$ increases. This could be expected because the level of turbulence increases. However, we can see that the

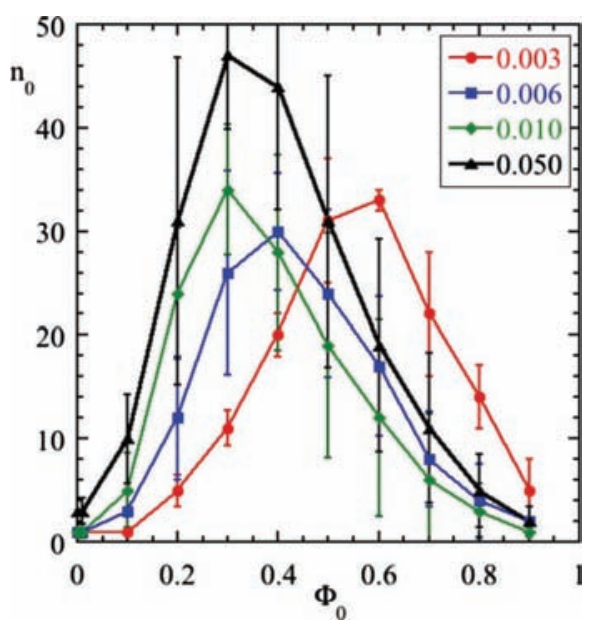

FIG. 14. (Color online) Number of large-scale components as a function of $\Phi_{0}$ for the four values of $\beta_{0}$ considered in this study. The values in this plot are the time-averaged values for each $\Phi_{0}$ and the error bars are the standard deviations. 


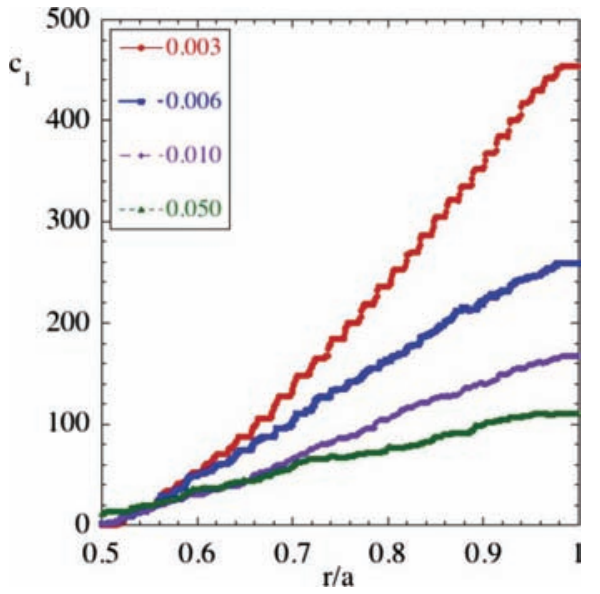

FIG. 15. (Color online) Number of large-scale cycles in the flow structure with $\Phi_{0}=0.1$ as a function of radius for the different values of $\beta_{0}$.

time variation reflected by the error bars is of the same order as the change with $\beta$.

For all $\beta$ values, we have also calculated $c_{1}(r)$, which is the number of large-scale cycles up to the radius $r$. The number of large-scale cycles is relatively uniformly distributed along the radius. For the different values of $\beta_{0}$, this is illustrated in Fig. 15. As $\beta$ increases, the total number of cycles decreases as we already seen in Fig. 11, but this decrease is relatively uniform along the radius. The largest component near the edge contains a number of these cycles.

By comparing the components with the number of largescale cycles as a function of the radius, we get a better picture of the flow structure. An example of such a comparison is done in Fig. 16 for one of the cases from Fig. 13, the one with $\Phi_{0}=0.4$. We can see that at the lowest value of the radius, the components do not have any cycles. For $r / a$ around 0.6, some of the components, which have broken away from the large one, have a few cycles. However, most of the cycles are on the large component and near the edge. On the outer region much of the structure seen at low $\beta$ has

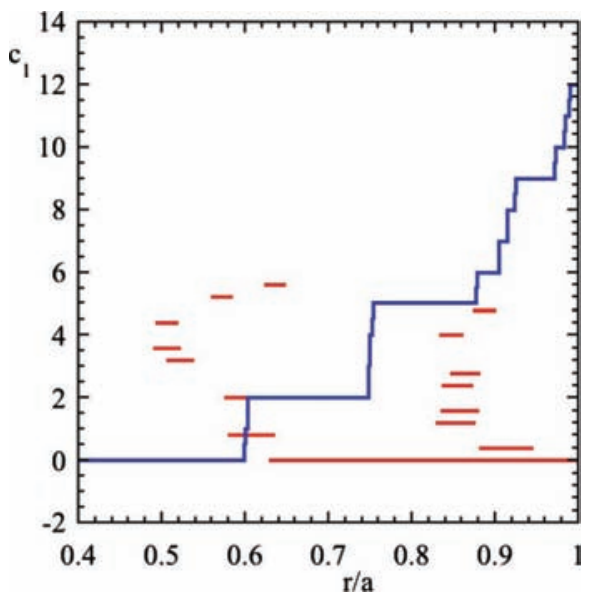

FIG. 16. (Color online) Large-scale components and $c_{1}(r)$ for $\beta=0.05$ and $\Phi_{0}=0.4$. The largest number of large-scale cycles is localized on the larger component near the plasma edge.

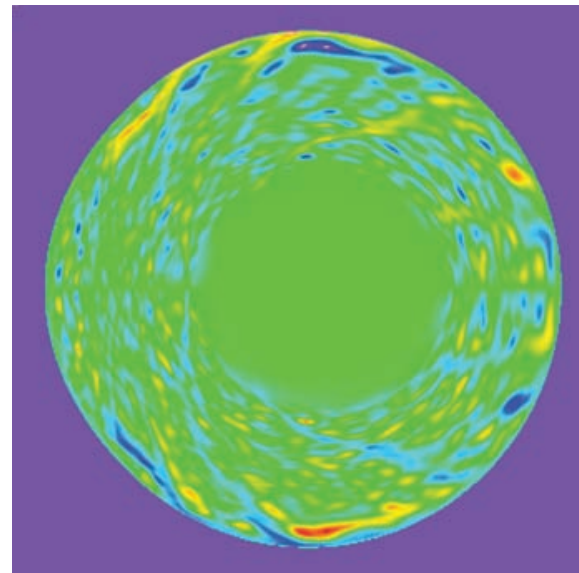

FIG. 17. (Color online) Contour plot of $\Phi$ on the toroidal cross section $\zeta=0$ at a given time for $\beta_{0}=0.05$.

survived at high $\beta$. This distribution of large-scale components and large-scale cycles is far from uniform and shows clearly the lack of homogeneity of the turbulence.

The presence of these large components is a clear indication that the turbulence is neither homogeneous nor isotropic even at the high $\beta$ value. This lack of homogeneity was not easy to detect in the two-dimensional (2D) contour plots of stream function. An example of such plots is shown in Fig. 17. This absence of homogeneity and isotropy has an impact in the particle transport. In Ref. [18], we already observed this lack of homogeneity as a dependence of the exponent of the algebraic tail of the distribution of particle flights on the poloidal position of the initial distribution of particles. We will look in more detail at the impact of the topology on transport in the next section.

\section{RELATION BETWEEN FLOW TOPOLOGY AND TRACER TRANSPORT}

One way to study the connection between topological properties of the flow and transport properties is by using the continuous time random walk (CTRW) approach [19]. The CTRW approach allows us to construct transport models based on statistical properties of the microscopic motion of the particles. Basically one assumes that the particle trajectories are composed by waiting times at a given position and particle flights between two different consecutive positions.

Let $\lambda(\Delta)$ be the probability that a particle performs a flight of length $\Delta$, and $\psi(\tau), \tau>0$, the probability that a particle waits a time $\tau$ between two consecutive flights. Montroll and Weiss [19] derived an evolution equation for the probability of finding a particle at point $x$ at time $t, P(x, t)$. Namely,

$$
\begin{aligned}
P(x, t)= & P_{0}(x)\left[1-\int_{0}^{t} \psi\left(t^{\prime}\right) d t^{\prime}\right] \\
& +\int_{0}^{t} \psi\left(t-t^{\prime}\right)\left[\int_{-\infty}^{\infty} \lambda\left(x-x^{\prime}\right) P\left(x^{\prime}, t^{\prime}\right) d x^{\prime}\right] d t^{\prime},
\end{aligned}
$$

where $P_{0}(x)$ is the initial condition at time $t=0$. This equa- 
tion is solved by using a Laplace-Fourier transform. The asymptotic behavior for $x$ and $t$ going to infinity gives the fluid limit of this equation. If in the large $t$ limit $\psi(t)$ is a decaying exponential and for the large $x$ limit of $\lambda(x)$ is a Gaussian, the fluid limit of the CTRW equation is the normal diffusion equation,

$$
\frac{\partial P}{\partial t}=D \frac{\partial^{2} P}{\partial x^{2}} .
$$

However, if in the large $t$ limit $\psi(t) \rightarrow t^{-(\beta+1)}$ and for the large $x$ limit $\lambda(x) \rightarrow|x|^{-(\alpha+1)}$, the fluid limit of the CTRW equation is a fractional differential equation

$$
\frac{\partial^{\beta} P}{\partial t^{\beta}}=D \frac{\partial^{\alpha} P}{\partial|x|^{\alpha}},
$$

where we use the conventional notation $(\alpha$ and $\beta$ ) for the indexes of the fractional derivatives in space and time. In the rest of the paper, the symbol $\beta$ is used for the ratio of the plasma pressure to the magnetic pressure.

For the definition of the fractional derivatives, see Ref. [8]. This different asymptotic behavior of the distribution functions changes fundamentally the nature of the transport and it is called anomalous diffusion.

In this section, we take a first step in connecting the topological properties of the turbulent flow considered in the last two sections with the properties of the particle tracer distribution. When we use the CTRW to interpret the transport results, we take the variable $x$ to be the radial variable. The tracer particle trajectories are the solution of the equation

$$
\frac{d \mathbf{r}}{d t}=\nabla \Phi \times \hat{\mathbf{b}}+V_{\|} \hat{\mathbf{b}}
$$

where we add a constant velocity $V_{\|}$along the magnetic field to the perpendicular velocity given by Eq. (1) in order for the tracers to explore the whole topology of the torus. More details on the tracer particle transport for the configurations for the resistive pressure-gradient-driven turbulence can be found in Ref. [18]. In this section, we analyze only the connection between transport properties and flow topology.

As the tracer particles move by the turbulent flow, we consider the particles being trapped for certain periods of times in the flow filaments and then taking steps or flights between trapping times. This way of looking at the tracer particle motion allows us to connect with the CTRW transport approach.

To understand the transport from the CTRW perspective, we identify the probability distribution function of the waiting times with the distribution function of the trapping times. As discussed in [10] and only for the quasicoherent flow structure, the existence of the filamentary structures induces an algebraic tail to the distribution of the trapping times. In absence of large-scale cycles, the distribution of trapping times has an exponential tail. As we commented above, the significance of this change is that power law leads to fractional derivative in time while the exponential to a normal first order derivative.

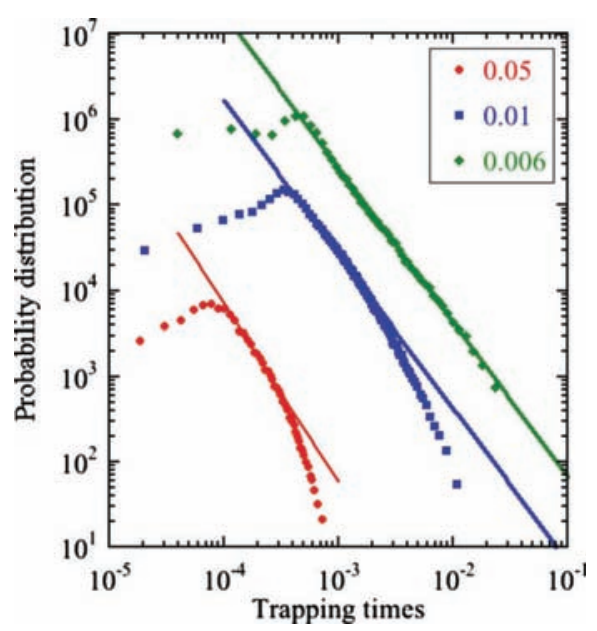

FIG. 18. (Color online) Distribution of trapping times for different $\beta_{0}$ values. In the figure is also shown the range of the algebraic tail for each individual case. To fit in the figure, the data for the $\beta_{0}=0.006$ has been multiplied by 1000 and for the $\beta_{0}=0.01$ by 100 .

To calculate the probability distribution of the trapping times and the flights we have to first define both of them in the context of our numerical calculations. There is not a unique way of defining particle flights. Here, we are interested in the radial transport and for this reason we consider flights only in the radial direction. We use the same definition as in Ref. [18]. We say that a particle tracer performs a flight while it moves on a trajectory keeping the same sign of the radial velocity.

Let us define the length of the flight $i$ in the radial direction as $\Delta \rho_{i}$. If a flight is very short, $\Delta \rho_{i} / a<0.005$, it means that the particle tracer is in a filament, we do not include it with the flights. We consider in this case that the tracer is trapped in a filament and we add the times of the consecutive short flights to calculate the trapping times. The value chosen for the upper bound of $\Delta \rho_{i}, 0.005 a$, corresponds to the smaller value of the different filament widths. In Ref. [10], we used an alternative definition for the trapping time. It was defined as the time that two particles initialized close together stick together. Both definitions lead to very similar trapping time distributions.

For the calculations discussed here, we can see that the range of the algebraic tail of the trapping time distribution is a function of $\beta$. In Fig. 18, we have plotted the distribution of trapping times for different $\beta_{0}$ values. As $\beta_{0}$ increases, the temporal range of the algebraic tail decreases significantly. This shows that a correlation exists between the number of large-scale cycles and the time range of the power-law region. For the high $\beta_{0}$ value, where the number of cycles is small, it is not clear that the power-law region even exists. Higher resolution calculations are needed to clarify this point. We do not have yet a quantitative relation between $c_{1}$ and this temporal range, but this problem is under study.

The fragmentation of the flow structure in components may have an impact on the length of the flights of the particles. In the previous section, we have described how fragmented the flow is. We can consider all the large-scale components of the flow for all values of $\Phi_{0}$. The distribution of 


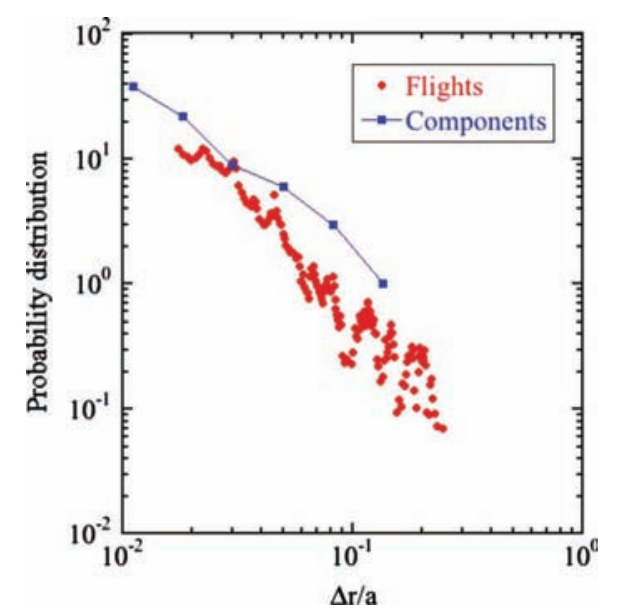

FIG. 19. (Color online) Distribution of the large-scale connected components and distribution of the particle flights at a given time for $\beta_{0}=0.01$.

the large-scale connected components can be compared to the distribution of the particle flights at a given time. That is, we calculate the flights for a given flow distribution without considering its time evolution. A comparison between these two distributions is shown in Fig. 19 for $\beta_{0}=0.01$. Both distributions have similar ranges and exponents for the power tail.

When the flights are calculated for a time evolving flow, the tail of the flights distribution has less oscillations that the one shown in Fig. 19, but the exponent of the algebraic fall off is somewhat larger, -2.2 compared to -1.7 . The reason is that there are other dynamical phenomena that contribute to the flights, like the occurrence of avalanches. This combination of a distribution of power-law steps with dynamically triggered avalanches has already been considered within the CTRW approach. In Refs. [20,21], a version of the CTRW model was introduced with Lévy distributions for the flights and a critical gradient condition to include the dynamical effects. This approach will be pursued further in order to relate the distribution of the large-scales components, which is effectively an eddy-size distribution, to the dynamics of the transport.

Therefore, the two topological properties of the turbulent flows considered in this paper, the large-scale cycles and large-scale components, provide the basis for the interpretation of some of the basic features of the turbulence-induced transport. They also show how the characteristic properties of the transport change as $\beta$ increases.

\section{CONCLUSIONS}

The number of large-scale cycles and the number of largescale connected components of the flow structure are a useful characterization of the topological properties of the turbulent flow in the resistive pressure-gradient-driven model. They are useful in characterizing the transition to the fully developed turbulence state. This is a quantitative characterization, which gives a better indication on how the flow transitions from single quasicoherent mode at low $\beta$ to a fully developed turbulence.
They are also useful in providing a connection between properties of the turbulence and properties of the induced transport. The number of large-scale cycles is directly related to the range of the algebraic tail of the particle trapping times. The distribution of the large-scale components gives the distribution of flights at a fixed time. These connections need to be pursued in a more quantitative manner in the future.

In Ref. [18], we concluded that the tracer particle transport in a $\beta_{0}$ range around 0.01 could be described by fractional differential equations. These equations change to normal diffusion at higher $\beta$. We see now that this change is due to the decrease of the large-scale cycles and to the increase in the number of large-scale connected components of the flow.

These topological parameters also reveal the presence of relatively large flow structures, which cannot be easily detected by pure visualization of the structure. The presence of relatively large components at high $\beta$ is an indication that the turbulence is not homogeneous at these $\beta$ values.

\section{ACKNOWLEDGMENTS}

We are grateful to the CHomP group for providing us with the software we needed to carry out the calculations presented here. In particular, we acknowledge very useful discussions with Marcio Gameiro, Pawel Pilarcyk, and Konstantin Mischaikow. Part of this research was sponsored by the Dirección General de Investigación of Spain under Project No. ENE2006-15244-C03-01. One of us (BAC) is grateful for the "Cátedra de Excelencia" from Universidad Carlos III and Banco Santander. The authors thankfully acknowledge the computer resources, technical expertise and assistance provided by the Barcelona Supercomputing Center-Centro Nacional de Supercomputación (http:// www.bsc.es).

\section{APPENDIX: CALCULATION OF THE NUMBER OF LARGE-SCALE CYCLES}

For resistive pressure gradient driven turbulence, each $\Phi_{m, N}$ component is localized at the radial position of its singular surface $\rho_{m}$. Because of the angular dependence associated with this Fourier component, this localization of the flow appears as a filamentary eddy twisting around the torus and closing on itself after $m$ toroidal turns for $N$ poloidal turns. On Fig. 4, we show a simple example for $N=3$. The filaments are toroidal knots on their own, if they are viewed as solid, their Betti numbers are simply $b_{0}=1, b_{1}=1$, and $b_{2}=0$.

There is one such filament in each rational surface, but they merge on the outer region of the torus. This happens because the magnetic field is weaker in the outer region of the torus and the flow filaments are thicker (ballooning effect). The region where the filaments merge are the so-called streamers [22]. Because they merge, they form a single connected component, so $b_{0}=1$, but the number of cycles they form is more complicated to evaluate. In Ref. [11] we have shown that this number is 


$$
b_{1}=1+N\left(m_{1}-m_{0}\right),
$$

where $m_{0}$ and $m_{1}$ are the minimum and maximum $m$ values in the structure, respectively. In this particular case $m_{0}=3$, $m_{1}=5$, and $b_{1}=7$.

In this simple example, the flow structure twists around the torus, and there are no other cycles than the large-scale cycles around the torus. However, when we cover the structure with cubes, some of them covering different filaments can touch and in this way they may create small false cycles. To get rid of these false cycles, we first notice that they are preserved when we cut the torus along the poloidal angle $\theta=\pi$ while the large scale cycles are broken. Let us call $X$ a cubical set approximating our structure and $A$ the result of cutting $X$ at $\theta=\pi$. Then, let $b_{0}[X], b_{1}[X]$ and $b_{2}[X]$ be the Betti numbers of $X$ and $b_{0}[A], b_{1}[A]$ and $b_{2}[A]$ the ones of $A$. In the situation just described, the number of (independent) cycles of $X$ minus the number of (independent) cycles of $A$,

$$
c_{1}=b_{1}[X]-b_{1}[A],
$$

is the number of (independent) large-scale cycles of our structure.

When we work with the cubical sets covering a structure, small false cycles may form, as we have already mentioned. Some of these cycles may meet the poloidal angle $\theta=\pi$ and so disappear as cycles of $A$. Therefore, the difference $b_{1}[X]-b_{1}[A]$ may be a bit greater than the number of cycles of our simplified structure. The specific method described here cannot be applied in general. For instance, it may happen that cutting the configuration creates new cycles. We need to develop an approach on the same lines described here but with broad validity.

Let us call $X$ a cubical set approximating our structure and $A$ the result of cutting $X$ at $\theta=\pi$. Let $b_{0}[X], b_{1}[X]$, and $b_{2}[X]$ be the Betti numbers of $X$ and $b_{0}[A], b_{1}[A]$ and $b_{2}[A]$ the Betti numbers of $A$.

In the general case, $c_{1}$ is greater than the difference $b_{1}[X]-b_{1}[A]$. For instance, some cubes may form a tube in $X$ near the poloidal angle $\theta=\pi$, and get cut in $A$ in two portions. If the tube is closed at one end, we will have a noncontractible loop in $A$ that is contractible as a loop in $X$. Figure 20(a) illustrates this phenomenon. So when we compute the difference $b_{1}[X]-b_{1}[A]$, we subtract a cycle of $A$ that was not taken into account in $b_{1}[X]$. If the tube is not closed at either end, then we will have two independent loops in $A$ that are not independent in $X$, as we can deform one into the other. In this case, we subtract two cycles of $A$ that correspond to just one in $X$. Figure 20(b) illustrates this phenomenon.

Therefore, the number $c_{1}$ we want to compute is of the form

$$
c_{1}=b_{1}[X]-b_{1}[A]+n_{1},
$$

where $n_{1}$ is the number of independent cycles of $A$ that cannot be contracted to a point in $A$ but can be contracted to a point in $X$ or can be deformed to another cycle in $X$.

For the computation of $c_{1}$ we shall use homology theory. Homology theory links a set of groups to each space $X$. These groups are the so-called homology groups and denoted (a)
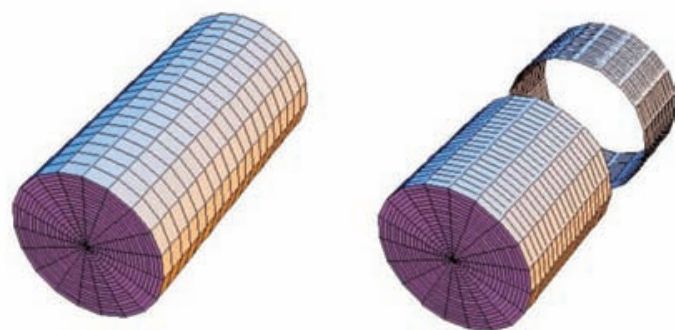

(b)
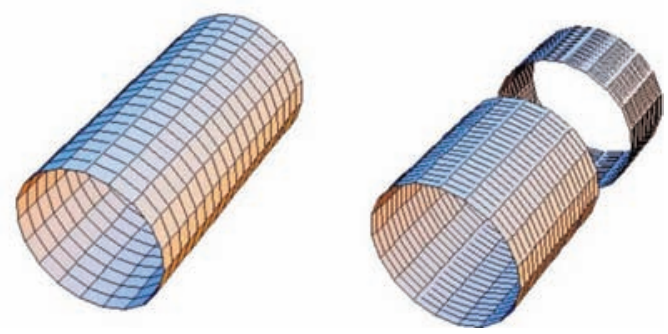

FIG. 20. (Color online) (a) Cutting a closed tube at one end generates a closed tube at one end and a cycle; (b) cutting a cycle generates two cycles.

by $H_{j}(X)$, for each integer $j \geq 0$. In our problem, $X$ is a subspace of $\mathbb{R}^{3}$ and $H_{j}(X)=0$, if $j \geq 3$. The first three groups are of the form

$$
H_{j}(X) \cong\left\{\left(i_{1}, \ldots, i_{b_{j}[X]}\right) \mid i_{1}, \ldots, i_{b_{j}[X]} \in \mathbb{Z}\right\}=\mathbb{Z}^{b_{j}[X]}, \quad j=0,1,2
$$

Here, the symbol $\cong$ denotes group isomorphism. The ranks of the homology goups $H_{j}(X), b_{j}[X]$, are called the Betti numbers of the space $X$. Their meaning is as follows:

$b_{0}[X]$ is the number of connected components of $X$. Each one of the basis elements $(1,0, \ldots, 0),(0,1, \ldots, 0), \ldots,(0,0, \ldots, 1) \in \mathbb{Z}^{b_{0}[X]}$ represents one of the connected components of $X$.

$b_{1}[X]$ is the number of independent cycles that cannot be contracted to one point in $X$. Each one of the basis elements $(1,0, \ldots, 0),(0,1, \ldots, 0), \ldots,(0,0, \ldots, 1) \in \mathbb{Z}^{b_{1}[X]}$ represents one of the cycles of $X$.

$b_{2}[X]$ is the number of independent cavities of $X$. Each one of the basis elements $(1,0, \ldots, 0),(0,1, \ldots, 0), \ldots,(0,0, \ldots, 1) \in \mathbb{Z}^{b_{2}[X]}$ represents one of the cavities of $X$.

On the other hand, homology theory also links to each pair of spaces, $A \subset X$, a set of groups $H_{j}(X, A)$ called relative homology groups of the pair $(X, A)$. For compact spaces of $\mathrm{R}^{3}$, all these groups are zero from the fourth onwards. The first four groups are

$$
H_{j}(X, A) \cong \mathbb{Z}^{b_{j}[X, A]}, \quad j=0,1,2,3 .
$$

The relative homology groups are related to the homology of two spaces by a sequence of morphisms, 


$$
\begin{aligned}
0 & \rightarrow H_{3}(X, A) \rightarrow H_{2}(A) \rightarrow H_{2}(X) \rightarrow H_{2}(X, A) \rightarrow H_{1}(A) \\
& \rightarrow H_{1}(X) \rightarrow H_{1}(X, A) \rightarrow H_{0}(A) \rightarrow H_{0}(X) \rightarrow H_{0}(X, A) \rightarrow 0
\end{aligned}
$$

that is an exact sequence, i.e., the image of each morphism is equal to the kernel of the next morphism.

The morphisms $H_{j}(A) \rightarrow H_{j}(X), j=0,1,2$ are induced by inclusion.

Recall that in Eq. (A3), we have denoted by $n_{1}$ the number of independent cycles of $A$ that cannot be contracted to a point in $A$ but can be contracted to a point in $X$ or can be deformed to another cycle in $X$. Those cycles generate a subgroup $\mathbb{Z}^{n_{1}} \subset H_{1}(A)$, that is precisely the kernel of the morphism $H_{1}(A) \rightarrow H_{1}(X)$. Therefore, from the sequence (A6) we get a sequence

$$
\begin{aligned}
0 & \rightarrow \mathbb{Z}^{n_{1}} \rightarrow H_{1}(A) \rightarrow H_{1}(X) \rightarrow H_{1}(X, A) \rightarrow H_{0}(A) \rightarrow H_{0}(X) \\
& \rightarrow H_{0}(X, A) \rightarrow 0
\end{aligned}
$$

that is also exact and this implies that the alternate sum of the ranks is zero,

$$
n_{1}-b_{1}[A]+b_{1}[X]-b_{1}[X, A]+b_{0}[A]-b_{0}[X]+b_{0}[X, A]=0 \text {. }
$$

Hence,

$$
c_{1}=b_{1}[X]-b_{1}[A]+n_{1}=b_{1}[X, A]-b_{0}[A]+b_{0}[X]-b_{0}[X, A] .
$$

In our case, all the components of $X$ have points of $A$, so that $b_{0}[X, A]=0$, and

$$
c_{1}=b_{1}[X, A]-b_{0}[A]+b_{0}[X] .
$$

Since the software CHomP also evaluates the relative homology, this formula can be used to compute $c_{1}$.

Notice that the relative homology is only defined when $A$ is a subspace of $X$. However, in the previous description, that was not the case. Actually, $A$ has the same number of cubes as $X$. In practice, we have slightly expanded the cubical space $X$ along $\theta=\pi$ by duplicating the set of cubes at this poloidal angle. This change does not alter the topology of $X$. The subspace $A$ is the result of eliminating this set of duplicated cubes.

The excision theorem [15] allows to calculate the relative homology of $(X, A)$ using subsets of $X$ and $A$ that are around the region of the $\theta=\pi$ cut. That implies that the cubical set used as data input can be reduced by a factor of $3 /\left(N_{\theta}+1\right)$. Therefore, we can go to higher resolutions in calculating $b_{1}[X, A]$.
[1] B. A. Carreras, V. E. Lynch, D. E. Newman, and G. M. Zaslavsky, Phys. Rev. E 60, 4770 (1999).

[2] B. A. Carreras, V. E. Lynch, L. Garcia, M. Edelman, and G. M. Zaslavsky, Chaos 13, 1175 (2003).

[3] D. del-Castillo-Negrete, B. A. Carreras, and V. E. Lynch, Phys. Rev. Lett. 94, 065003 (2005).

[4] R. Sanchez, B. A. Carreras, D. E. Newman, V. E. Lynch, and B. Ph. van Milligen, Phys. Rev. E 74, 016305 (2006).

[5] I. Calvo, R. Sanchez, B. A. Carreras, and B. Ph. van Milligen, Phys. Rev. Lett. 99, 230603 (2007).

[6] J. A. Mier, R. Sanchez, L. Garcia, B. A. Carreras, and D. E. Newman, Phys. Rev. Lett. 101, 165001 (2008).

[7] R. Sanchez, D. E. Newman, J.-N. Leboeuf, B. A. Carreras, and V. Decyk, Phys. Plasmas 16, 055905 (2009).

[8] I. Podlubny, Fractional Differential Equations (Academic, San Diego, 1999).

[9] L. Garcia, B. A. Carreras, and V. E. Lynch, Phys. Plasmas 9, 47 (2002).

[10] G. M. Zaslavsky, B. A. Carreras, V. E. Lynch, L. Garcia, and M. Edelman, Phys. Rev. E 72, 026227 (2005).

[11] B. A. Carreras, I. Llerena, L. Garcia, and I. Calvo, Phys. Rev.
E 78, 066402 (2008).

[12] http://chomp.rutgers.edu

[13] T. Kaczynski, K. Mischaikow, and M. Mrozek, Computational Homology (Springer, Berlin, 2004).

[14] I. Calvo, L. Garcia, B. A. Carreras, R. Sanchez, and B. Ph. van Milligen, Phys. Plasmas 15, 042302 (2008).

[15] J. M. Munkres, Elements of Algebraic Topology (AddisonWesley, Reading, MA, 1984).

[16] H. R. Strauss, Phys. Fluids 20, 1354 (1977).

[17] J. F. Drake and T. M. Antonsen, Jr., Phys. Fluids 27, 898 (1984).

[18] L. Garcia and B. A. Carreras, Phys. Plasmas 13, 022310 (2006).

[19] E. W. Montroll and G. H. Weiss, J. Math. Phys. 6, 167 (1965).

[20] B. Ph. van Milligen, R. Sanchez, and B. A. Carreras, Phys. Plasmas 11, 2272 (2004).

[21] R. Sanchez, B. Ph. van Milligen, and B. A. Carreras, Phys. Plasmas 12, 056105 (2005).

[22] P. Beyer, S. Benkadda, X. Garbet, and P. H. Diamond, Phys. Rev. Lett. 85, 4892 (2000). 